NBI-HE-96-64

TIT/HEP-352

November 1996

\title{
Quantum geometry of topological gravity
}

\author{
J. Ambjørn ${ }^{1}$, K. N. Anagnostopoulos ${ }^{1}$, T. Ichihara ${ }^{2}$, L. Jensen ${ }^{1}$, \\ N. Kawamoto ${ }^{3}, Y$. Watabiki ${ }^{2}$ and K. Yotsuji ${ }^{3}$ \\ ${ }^{1}$ The Niels Bohr Institute, University of Copenhagen, \\ Blegdamsvej 17, DK-2100 Copenhagen Ø, Denmark \\ ${ }^{2}$ Department of Physics, Tokyo Institute of Technology, \\ Ø-okayama, Meguro, Tokyo 152, Japan \\ ${ }^{3}$ Department of Physics, Hokkaido University, \\ Sapporo, Japan
}

\begin{abstract}
We study a $c=-2$ conformal field theory coupled to two-dimensional quantum gravity by means of dynamical triangulations. We define the geodesic distance $r$ on the triangulated surface with $N$ triangles, and show that $\operatorname{dim}\left[r^{d_{H}}\right]=\operatorname{dim}[N]$, where the fractal dimension $d_{H}=3.58 \pm 0.04$. This result lends support to the conjecture $d_{H}=-2 \alpha_{1} / \alpha_{-1}$, where $\alpha_{-n}$ is the gravitational dressing exponent of a spin-less primary field of conformal weight $(n+1, n+1)$, and it disfavors the alternative prediction $d_{H}=-2 / \gamma_{\text {str }}$. On the other hand, we find $\operatorname{dim}[l]=\operatorname{dim}\left[r^{2}\right]$ with good accuracy, where $l$ is the length of one of the boundaries of a circle with (geodesic) radius $r$, i.e. the length $l$ has an anomalous dimension relative to the area of the surface. It is further shown that the spectral dimension $d_{s}=1.980 \pm 0.014$ for the ensemble of (triangulated) manifolds used. The results are derived using finite size scaling and a very efficient recursive sampling technique known previously to work well for $c=-2$.
\end{abstract}




\section{Introduction}

Liouville theory and matrix models have been successful in explaining a number of features of conformal field theories coupled to two-dimensional quantum gravity. However, our primary interest in a theory of quantum gravity concerns geometry: which concepts of geometry survive the quantum average, and how is the geometry changed by this average. These questions have not been clarified by Liouville field theory or matrix model techniques. In the last couple of years significant progress has been made in this direction, starting with the introduction of the so-called transfer matrix [1]. It was shown that a reparametrization invariant formulation of the twopoint function in quantum gravity has a simple geometric interpretation [2] and that a generalization of the two-point function to include matter fields allows a geometric interpretation of the KPZ-exponents [3], an interpretation first conjectured in [4]. In addition it was realized that finite size scaling analysis of the two-point functions were very efficient tools for extracting critical exponents [5, 4. In this article we will take advantage of this new technology and combine it with the efficient recursive sampling algorithm developed earlier for $c=-2$ conformal field theory coupled to two-dimensional quantum gravity used in [6] where the fractal nature of quantum gravity in two dimensions was first numerically confirmed.

\section{The model}

The $c=-2$ model coupled to quantum gravity corresponds to a non-unitary $(1,2)$ conformal field theory coupled to quantum gravity, known as a topological quantum gravity. There exists an explicit realization of the model within the framework of dynamical triangulations. In this framework the partition function for $c$ Gaussian fields coupled to two-dimensional quantum gravity is

$$
Z_{N}=\sum_{\mathcal{T}_{N}}\left(\operatorname{det} C_{\mathcal{T}_{N}}\right)^{-c / 2}
$$

In (四) the summation is over all triangulations $\mathcal{T}_{N}$ with fixed topology (which we will always assume to be spherical in this paper) built from $N$ triangles, and $C_{\mathcal{T}_{N}}$ is the socalled adjacency matrix of the graph corresponding to the triangulation $\mathcal{T}_{N}$. Notice that the triangulations of the spherical surface are in one-to-one correspondence with the $\phi^{3}$ connected planar graphs with no external legs, and thus it is possible to generate any triangulation $\mathcal{T}_{N}$ if we generate and connect $\phi^{3}$ trees and rainbow diagrams with the correct weight. If $c=-2$, the weight for generating $\phi^{3}$ trees and rainbow diagrams is 1, i.e. Eq. (11) can be written [7]

$$
Z_{N}=\frac{1}{N+2}\left(\sum_{\substack{\text { tree diagrams } \\ \text { with } N+2 \text { legs }}} 1\right)\left(\sum_{\substack{\text { rainbow diagrams } \\ \text { with }(N+2) / 2 \text { lines }}} 1\right), \quad(\text { for } c=-2),
$$

where the first summation is over all rooted $\phi^{3}$ tree diagrams with $N+2$ external legs and the second summation is over all rainbow diagrams with $(N+2) / 2$ lines. 
$1 /(N+2)$ is the symmetry factor which comes from connecting the tree diagram and the rainbow diagram. Using a recursive algorithm for generating $\phi^{3}$ trees and the rainbow diagrams in (21), it is possible to create a large number of independent triangulations $\mathcal{T}_{N}$ with the weight $\operatorname{det} C_{\mathcal{T}_{N}}$. We refer to [6] and [8] for details. Supplementary to the first studied in [6] we will in this work study the finite size aspects of the observables associated with $Z_{N}$, the main motivation being that finite size scaling by far is the most reliable method for extracting continuum physics in critical systems and we now understand that this is true also for these systems coupled to quantum gravity.

One very important point in the above setup is that we have the concept of distance, even if we usually associate $c=-2$ model with a topological gravity. To a triangulation $\mathcal{T}_{N}$ we can unambiguously associate a piecewise linear manifold with a metric dictated by the length assignment $\varepsilon$ to each link. From a practical point of view we use instead a graph-theoretical distance between vertices, links or triangles. In the limit of very large triangulations we expect that the different distances when used in ensemble averages will be proportional to each other. To be specific we will in the following operate with a "link distance" and a "triangle distance". The link distance between two vertices is defined as the shortest link-path between the two vertices, while the triangle distance between two triangles is defined as the shortest path along neighboring triangles between the two triangles. In this way the triangle distance becomes the link distance in the dual $\phi^{3}$ graph.

In the following we will report on the measurement of two quantities related to the fractal structure of quantum space-time: The total length $\langle l\rangle$ (and the higher moments $\left\langle l^{n}\right\rangle$ ) of boundaries of spherical balls of (geodesic) distance $r$, and the measurement of so-called spectral quantities, originating from the study of random walks on the manifolds.

Let us define the observables on the triangulation $\mathcal{T}_{N}$ more precisely. We consider a spherical ball of radius $r$ and its shell for a given triangulation $\mathcal{T}_{N}$. The spherical ball consists of all vertices with link distance $r^{\prime} \leq r$ and the spherical shell consists of all vertices with link distance $r$, where the distance is measured from a given vertex $v_{0}$ which is considered as the center of the spherical ball. In the same way we can define the spherical shell in terms of triangle distance. We will use both definitions in the following. The spherical shell will in general consists of a number of connected components. If we take the average over all positions of $v_{0}$ and all triangulations $\mathcal{T}_{N}$, we get a distribution $\rho_{N}(l, r)$ of the length $l$ (measured in link units) of the connected components of the spherical shells of radius $r$, i.e.

$$
\left\langle l^{n}\right\rangle_{r, N} \equiv \sum_{l=1}^{\infty} l^{n} \rho_{N}(l, r) .
$$

In particular we introduce the special notation $n_{N}(r)=\langle l(r)\rangle_{N}$ and expect the fractal dimension to be related to $n_{N}(r)$ by

$$
n_{N}(r) \sim r^{d_{H}-1}, \quad 1 \ll r \ll N^{1 / d_{H}} .
$$

According to general scaling arguments [2, 5, 团 we expect the following behaviour 
for $n_{N}(r)$ :

$$
n_{N}(r) \sim N^{1-1 / d_{H}} F_{1}(x), \quad x=\frac{r}{N^{1 / d_{H}}},
$$

and we expect $F_{1}(x)$ to fall off rapidly when $x \gg 1$.

The spectral properties are derived from the study of random walks on the triangulated surfaces via the diffusion equation

$$
\phi(v, t+1)=\frac{1}{n_{v}} \sum_{\left(v v^{\prime}\right)} \phi\left(v^{\prime}, t\right),
$$

where $t$ is the diffusion time and the summation is over the $n_{v}$ neighboring vertices' $v^{\prime}$ to $v$. We here consider the initial condition of $\phi(v, t)$,

$$
\phi(v, 0)=\frac{1}{n_{v}} \delta_{v, v_{0}},
$$

where $v_{0}$ is a fixed vertex. Eqs. (6) and (7) are discretizations of the diffusion equation on a continuum manifold. We will be interested in observables associated with the diffusion process obtained by averaging over the chosen vertex $v_{0}$ as well as different triangulations $\mathcal{T}_{N}$. In the following discussion we will always assume that this average has been performed. Let us denote the probability of diffusion out to a link distance $r$ in time $t$ by $k_{N}(r, t)$. By definition we have

$$
\sum_{r=0}^{\infty} n_{N}(r) k_{N}(r, t)=1
$$

From scaling arguments [9, 1, 10] we expect the following scaling

$$
k_{N}(r, t)=\frac{1}{N} p(x, y), \quad x=\frac{r}{N^{1 / d_{H}}}, \quad y=\frac{t}{N^{\lambda}},
$$

where the new exponent $\lambda$ is defined such that $y$ will be finite in the scaling limit. The spectral dimension is defined from the return probability by

$$
k_{N}(0, t) \sim \frac{N^{\lambda d_{s} / 2-1}}{t^{d_{s} / 2}}, \quad \text { for } \quad t \sim 0
$$

while the average geodesic distance travelled by diffusion at time $t$ is

$$
\langle r(t)\rangle_{N} \equiv \sum_{r=0}^{\infty} r n_{N}(r) k_{N}(r, t) \sim N^{1 / d_{H}-\lambda \sigma} t^{\sigma}, \quad \text { for } \quad t \sim 0 .
$$

If a kind of "smooth" fractal is expected here again, $k_{N}(0, t)$ and $\langle r(t)\rangle_{N}$ exist and are different from zero in the limit $N \rightarrow \infty$. This implies the scaling relations

$$
d_{s}=\frac{2}{\lambda}, \quad \sigma=\frac{1}{\lambda d_{H}}
$$

\footnotetext{
${ }^{1}$ Also in this case we have the possibility to formulate a diffusion in terms of triangles and triangle distances, rather than vertices and link distances. However, the finite size effects are larger for triangles and we will in the following only use link distances when we discuss diffusion.
} 


\section{$3 \quad$ Numerical results}

\subsection{The simulations}

The simulations are performed by generating a number of statistically independent configurations using the algorithm mentioned in the introduction (see [6] and [8] for details). We use the high quality random number generator RANLUX [16, 17] whose excellent statistical properties are due to its close relation to the Kolmogorov K-system originally proposed by Savvidy et.al. [14, 15] in 1986]. We report results on system sizes ranging from 2000-256000 triangles. The number of configurations obtained depends on the lattice size and on the observable that we measure. We choose 20 random vertices on each configuration in order to perform correlation function measurements. We need to collect more statistics to test Eq. (5), where we have between $4.2 \times 10^{6}$ and $1.6 \times 10^{6}$ configurations. For the $128 \mathrm{~K}$ and $256 \mathrm{~K}$ lattices we have $6 \times 10^{5}$ and $2 \times 10^{5}$ configurations respectively. In order to measure the moments $\left\langle l^{n}\right\rangle_{r, N}$ and their scaling properties we need a factor of $10^{2}$ less configurations: We have approximately 50000 configurations for each lattice size. Unfortunately, the computer effort for making the measurements is comparable to the one needed to test Eq. (5) with enough accuracy. For the diffusion equation we collect 2500 configurations on which we perform 5 measurements. For the three largest lattices we have 620,600 and 400 configurations respectively.

\subsection{The fractal dimension}

We have measured the fractal dimension in a number of independent ways 5 , and various measurements agree. Here we limit ourself to report on one particular method, based on the distribution $n_{N}(r)$. From (5) we have

$$
\langle r\rangle_{N} \equiv \frac{1}{N} \sum_{r=0}^{\infty} r n_{N}(r) \sim N^{1 / d_{H}} .
$$

Obviously, (13) could itself serve as a natural definition of $d_{H}$. By measuring $n_{N}(r)$ we can record $\langle r\rangle_{N}$ as a function of $N$ and hence determine $d_{H}$. Strictly speaking, we expect this relation to be valid in the limit of infinite $N$, while finite $N$ effects will be present in (13). The finite $N$ effects should be dictated by the "the number of points" $L$ corresponding to the linear size of the system, i.e. we expect

$$
\frac{\langle r\rangle_{N}}{N^{1 / d_{H}}}=\text { const. }+\frac{\text { const. }}{L}+\frac{\text { const. }}{L^{2}}+\cdots .
$$

\footnotetext{
${ }^{2}$ The history of the seminal paper by Savvidy et.al. is interesting: The paper was rejected by 4 computer journals, including Comput. Phys. Commun. where M. Lüscher finally published a related paper and F. James the FORTRAN code of RANLUX in 1994. These ideas were communicated to F. James by G.K. Savvidy during his stay at CERN in 1987.

${ }^{3}$ The details of these measurements will be reported elsewhere [8].
} 


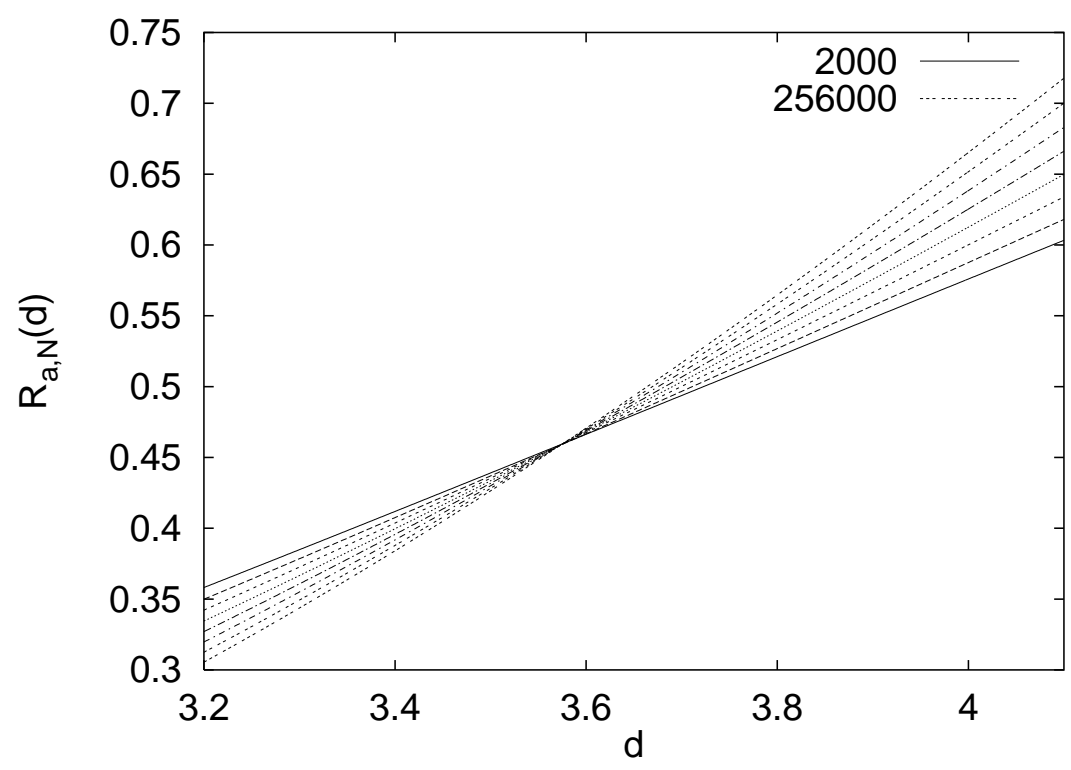

Figure 1: The functions $R_{a, N}(d)$ for $N=2 K, 4 K, 8 K, \ldots, 256 K$ and for the optimal $a=0.130$ determined by minimizing Eq. (19).

If we use the fact that $N^{1 / d_{H}}$ is a typical measure for the linear extension of the manifold, Eq. (14) can be written,

$$
\langle r+a\rangle_{N} \sim N^{1 / d_{H}}+O\left(\frac{1}{N^{1 / d_{H}}}\right)
$$

by identifying $L=N^{1 / d_{H}}$. The parameter $a$, which is considered the shift in $r$, incorporates the next order correction. We will discuss possible physical interpretations of the shift $a$ in detail in [8]. Now, let us define

$$
R_{a, N}(d)=\frac{\langle r+a\rangle_{N}}{N^{1 / d}}
$$

We determine the value of $a$ and $d_{H}$ in the following way: first we measure $\langle r\rangle_{N_{i}}$ for a certain number of different volumes $N_{i}$ of the universes, ranging from $N=2 K$ to $N=256 K$. For a given $a$ we choose, for each couple $N_{i}, N_{j}$ of $N$ 's, the $d_{H}^{i j}$ such that

$$
R_{a, N_{i}}\left(d_{H}^{i j}\right)=R_{a, N_{j}}\left(d_{H}^{i j}\right) .
$$

For this choice of $N_{i}, N_{j}$ we bin the data and estimate an error $\delta d_{H}^{i j}$. Then we determine the average

$$
\bar{d}_{H}=\frac{1}{\# \text { pairs }} \sum_{i \neq j} d_{H}^{i j},
$$

and compute

$$
\chi^{2}(a)=\sum_{i \neq j} \frac{\left(d_{H}^{i j}-\bar{d}_{H}\right)^{2}}{\left(\delta d_{H}^{i j}\right)^{2}} .
$$




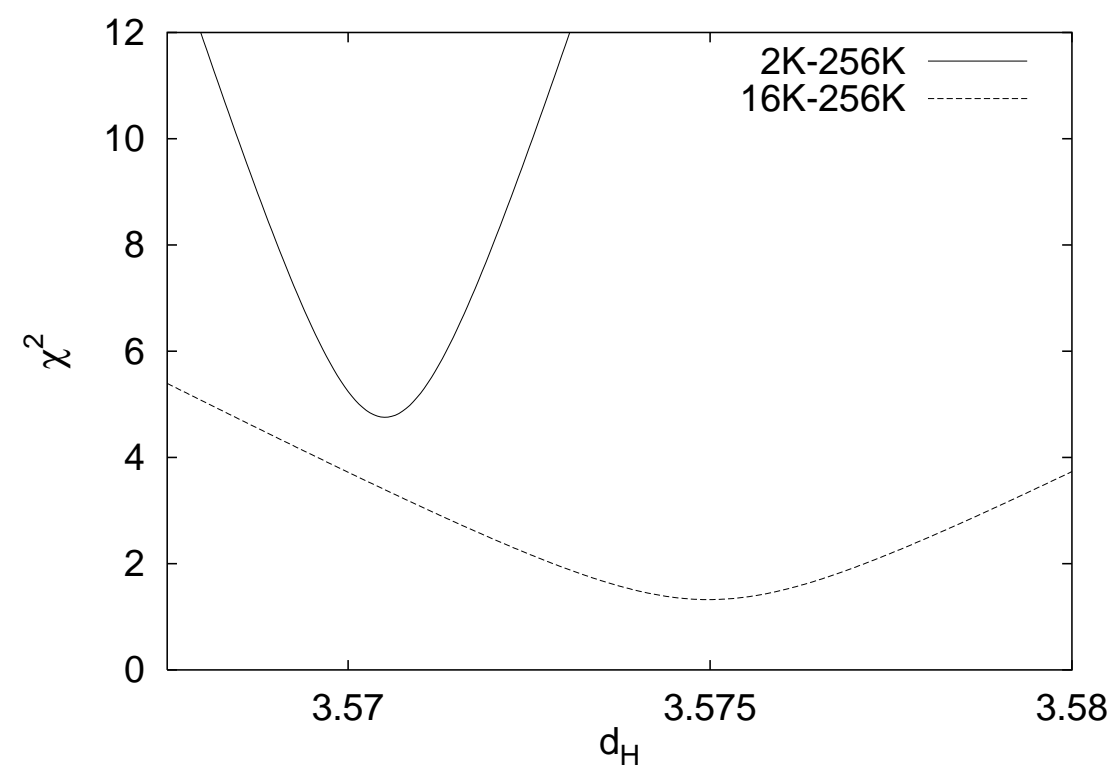

Figure 2: $\chi^{2}(a)$, defined by Eq. (19) for two sets of $N_{i}$ 's: $N_{i}=2 K, 4 K, \ldots, 256 K$ (solid curve) and $N_{i}=16 K, 32 K, \ldots, 256 K$ (dashed curve).

The preferred pair $\left(a, d_{H}(a)\right)$ is determined by the minimum of $\chi^{2}(a)$. This method works quite impressively. In Fig. 1 we have shown the intersection of the curves $R_{a, N}(d)$ as a function of $d$ for the optimal choice of $a$. The important point is that there exists a value of $a$ where the curves intersect with high precision and that the the range of $a$ where $\chi^{2}(a)$ is acceptably small, i.e. $O(1)$, is quite small and hence $d_{H}$ will be determined with high precision. In Fig. 2 we show $\chi^{2}(a)$. In this way we get

$$
a_{m}=0.139 \pm 0.005, \quad d_{H}\left(a_{m}\right)=3.574 \pm 0.003
$$

In (20) we have estimated the error as follows. Define an interval of acceptance $\left[a_{\min }, a_{\max }\right]$ of $a$ by demanding that $\chi^{2}(a)<2 \chi^{*}$ where $\chi^{*}=\max \left\{1, \chi^{2}\left(a_{m}\right)\right\}$ and find the variation of $d(a)$ in this interval. After this we repeat the whole procedure by making various cuts in the pairs of $N_{i}$ 's included in (18) and (19), discarding successively the smallest $N_{i}$ 's. The value of $d_{H}$ in Eq. (20) agrees with the original value $d_{H}=3.5 \pm 0.2$ [6]. The strength of finite-size scaling is that one can obtain higher precision results with the use of much smaller lattices. The original simulation needed $5000 K$ size lattices.

\subsection{The boundary}

We now turn to the measurements of $\left\langle l^{n}\right\rangle_{r, N}$. These observables are constructed from $\rho_{N}(l, r)$, which can readily be measured in the simulations. If $\operatorname{dim}[N]=\operatorname{dim}\left[l^{2}\right]$, then from scaling arguments, we expect

$$
\left\langle l^{n}\right\rangle_{r, N} \sim N^{n / 2} \tilde{F}_{n}(x), \quad x=\frac{r}{N^{1 / d_{H}}} .
$$



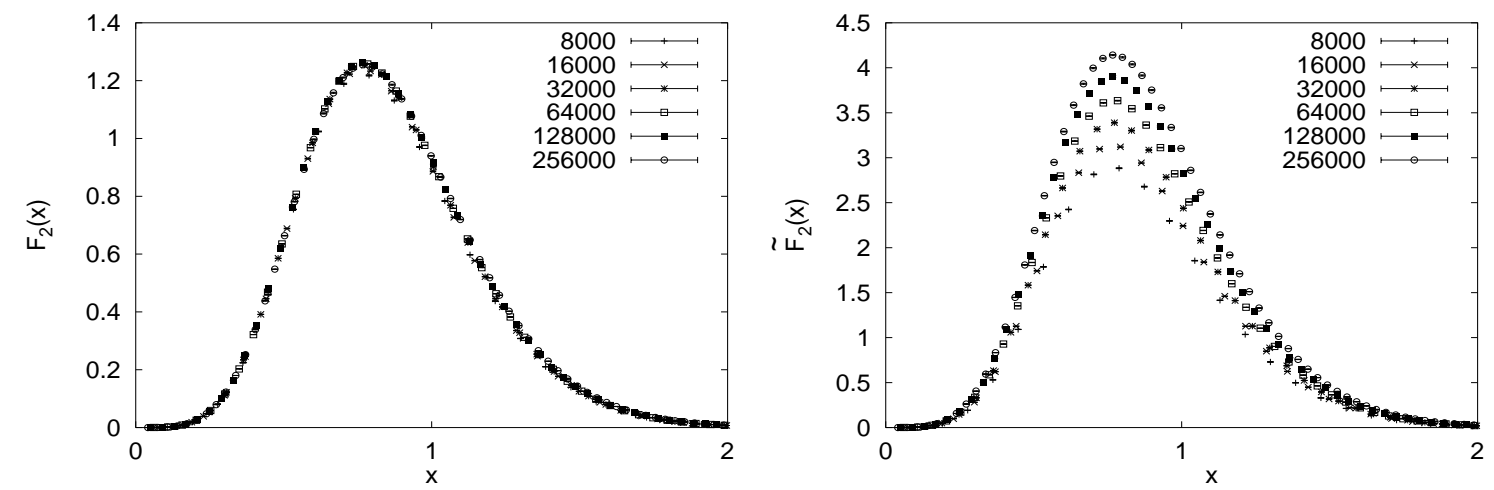

Figure 3: The scaling functions $F_{n}(x)$ defined by Eq. (22) (left figure) and the scaling functions $\tilde{F}_{n}(x)$ defined by Eq. (21), for $n=2$ and $N=8 K, 16 K, \ldots, 256 K$.

However, our measurements are consistent with the following scaling relation

$$
\left\langle l^{n}\right\rangle_{r, N} \sim N^{2 n / d_{H}} F_{n}(x), \quad \text { for } n \geq 2
$$

which implies that $\operatorname{dim}[l]=\operatorname{dim}\left[r^{2}\right]$. Eq. (22) indicates that we have

$$
\left\langle l^{n}\right\rangle_{r, N} \sim r^{2 n} \quad \text { for } 1 \ll r \ll N^{1 / d_{H}}, n \geq 2 .
$$

Again, relations like (22) are expected to be valid up to finite size effects, as in Eq. (14). As a first phenomenological correction we use a shift $r \rightarrow r+a$ as in (15) to find the best scaling function $F_{n}(x)$ for a suitable range of $N_{i}$ 's. In Fig. 3 we have shown $F_{n}(x)$ for $n=2$ for the values of $a$ which provide the best scaling function. Similar pictures exist for $n=3$ and $n=4$. This is to be compared to the scaling given in Eq. (21). We see that it is not possible to find a scaling function $\tilde{F}_{n}(x)$ if we used the ansatz (21). Assuming the scaling (22) we get an independent determination of $d_{H}=3.63 \pm 0.04$. This result is remarkably consistent with the value of $d_{H}$ determined by the other methods we used, considering the systematic errors due to finite size effects. One, however, should consider the possibility of a dimensional relation of the form:

$$
\operatorname{dim}[l]=\operatorname{dim}\left[r^{2(1-\epsilon)}\right]
$$

In this case, for given $d_{H}$, one can use the relation $\left\langle l^{n}\right\rangle_{r, N}=N^{2 n(1-\epsilon) / d_{H}} F_{n}(x)$ in order to determine the value of $\epsilon$. Using the lowest value we obtained for $d_{H}$ using other methods, we obtain $\epsilon<0.03$. Details will be published elsewhere [8].

\subsection{The spectral dimension}

Let us finally turn to the spectral dimension $d_{s}$, as defined by (10). For a given triangulation $k_{N}(0, t)$ can be calculated by means of (6) and (7), and the quantum average is then obtained by performing the average over different triangulations, since these are generated with the correct weight by the recursive sampling. In Fig. 


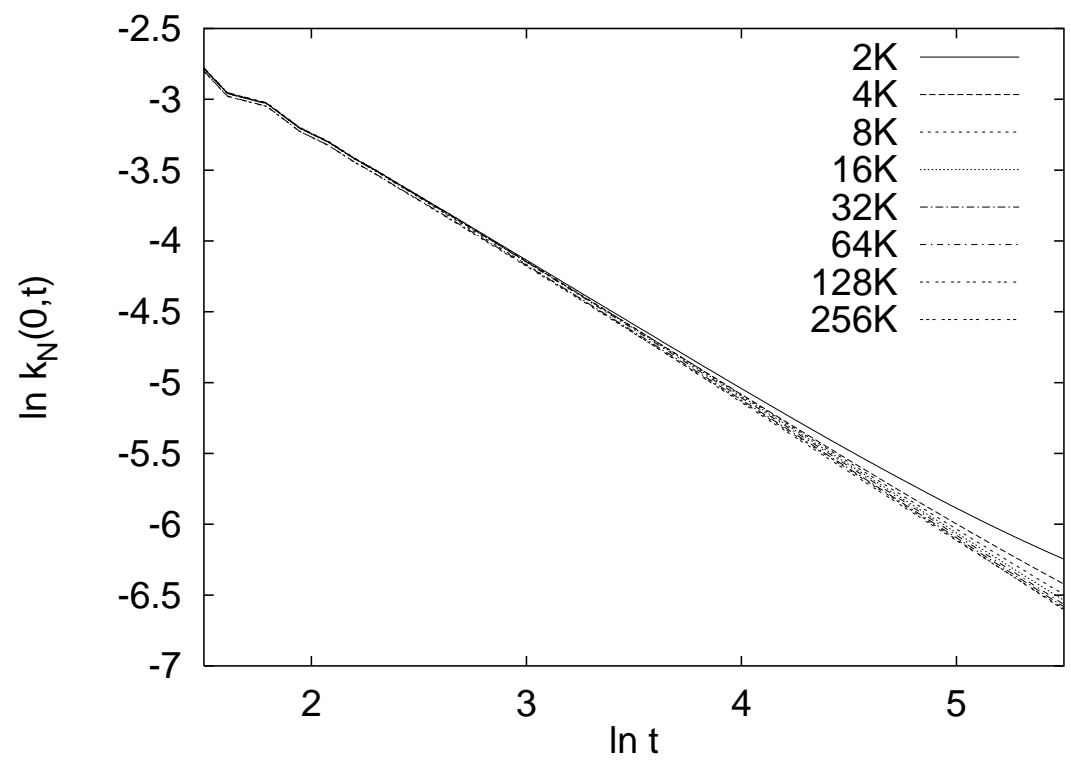

Figure 4: A plot of $\ln k_{N}(0, t)$ versus $\ln t$ for $N=2 K, 4 K, \ldots, 256 K$.

4 we have shown a plot of $\ln k_{N}(0, t)$ versus $\ln t$. When $t$ is too small, discretization effects interfere with the form (10), as discussed in details in [4]. For larger values of $t$ it is possible to perform a very good fit to (10), as is clear from Fig. 4 . For the $256 K$ lattice the value of $d_{s}$ is

$$
d_{s}=1.980 \pm 0.014
$$

Due to finite size effects, the value of $d_{s}$ depends on the lattice size, but it clearly approaches 2 as $N$ becomes large. Exploiting the $N$ dependence of $k_{N}(r, t)$ it is possible to determine the exponents $\lambda$ and $\sigma$ introduced in Eqs. (10) and (11). We find good agreement with the scaling prediction (12). A detailed analysis of the diffusion equation and the numerical verification of (12) will be published elsewhere.

\section{Discussion}

The fractal structure of quantum gravity coupled to matter has provided us with somewhat of a puzzle. The first analytic result suggested that [11]

$$
d_{H}=-\frac{2}{\gamma_{\mathrm{str}}}, \quad \gamma_{\mathrm{str}}=\frac{1}{12}(c-1-\sqrt{(25-c)(1-c)}) .
$$

This formula has later been obtained in a number of different ways using the stringfield approach developed in [12]. There are several problems with the "proofs". For instance, the string-field proof is based on the identification of proper time with geodesic distance. However, only in the case of pure two-dimensional gravity one can clearly identify the proper time used in string-field theory with the geodesic distance. Further, the formula (26) predicts that $d_{H} \rightarrow \infty$ for $c \rightarrow 1$ and $d_{H} \rightarrow 0$ for 
$c \rightarrow-\infty$. The latter result is clearly undesirable since one expects that $d_{H} \rightarrow 2$ in the semi-classical limit $c \rightarrow-\infty$. In fact (26) predicts $d_{H}=2$ in the case considered here, $c=-2\left(\gamma_{\mathrm{str}}=-1\right)$, and a very recent constructive proof, using an explicitly constructed transfer matrix for $c=-2$, confirms this prediction [13]. The prediction is clearly in disagreement with the computer measurements reported in this article. In a similar way the prediction (26) for $c>0$ has been in contradiction with the Monte Carlo simulations performed for the Ising model $\left(c=1 / 2, \gamma_{\mathrm{str}}=-1 / 3\right)$ and the three-states Potts model $\left(c=4 / 5, \gamma_{\text {str }}=-1 / 5\right)$. In these cases one could argue that since the fractal dimension predicted is so large, the systems used in the computer simulations have been too small to observe the correct fractal dimensions. Although it is hard to understand how one can get all predicted KPZ-exponents correct in the numerical simulations, but not being able to measure the fractal dimension, one could not rule out entirely this criticism. The important point is that the criticism is not valid for the present numerical investigation, since the predicted $d_{H}$ is small (namely 2 ), and we are able to deal with very large systems.

The alternative prediction [9] for $d_{H}$ is

$$
d_{H}=-2 \frac{\alpha_{1}}{\alpha_{-1}}=2 \times \frac{\sqrt{25-c}+\sqrt{49-c}}{\sqrt{25-c}+\sqrt{1-c}} .
$$

The origin of this equation is to be found in the analysis of the diffusion equation in Liouville theory [9] and is based on the observation that (8)-(12) imply that

$$
\operatorname{dim}\left[\left\langle r^{2}(t)\right\rangle_{N}\right]=\operatorname{dim}\left[N^{2 / d_{H}}\right] .
$$

This follows from the assumed scaling, independent of the specific model of dynamical triangulations. On the other hand, in Liouville theory one can use the De-Witt short distance expansion of the heat kernel in terms of geodesic distance to deduce [9] that

$$
\operatorname{dim}\left[\left\langle r^{2}(t)\right\rangle_{N}\right]=\operatorname{dim}\left[N^{-\left(\alpha_{-1} / \alpha_{1}\right)}\right]
$$

provided $r, N$ and $t$ are viewed as continuum geodesic distance, continuum area and continuum diffusion time in Liouville theory. In (29) $\alpha_{-n}$ denotes the gravitational dressing of a $(n+1, n+1)$ conformal field, i.e.

$$
\int d^{2} \xi \sqrt{g} \Phi_{n+1}(g) \rightarrow \int d^{2} \xi \sqrt{\hat{g}} e^{\alpha_{-n} \phi} \Phi_{n+1}(\hat{g}), \quad \text { for } \quad g_{\mu \nu}(\xi)=e^{\phi(\xi)} \hat{g}_{\mu \nu}(\xi)
$$

where $\hat{g}_{\mu \nu}(\xi)$ is the background metric. The requirement that $e^{\alpha_{-n} \phi} \Phi_{n+1}(\hat{g})$ is a $(1,1)$ conformal field fixes

$$
\alpha_{n}=\frac{2 n}{1+\sqrt{(25-c-24 n) /(25-c)}} .
$$

For $c=0$ one obtains $d_{H}=4$ (in agreement with the transfer matrix prediction), while for $c=-2$ the prediction is

$$
d_{H}(c=-2)=(3+\sqrt{17}) / 2=3.561 \ldots .
$$


It agrees with the numerical results reported in this paper.

However, clearly this is not the complete story since we have also obtained

$$
\operatorname{dim}\left[l^{n}\right]=\operatorname{dim}\left[r^{2 n}\right], \quad \text { for } n>1 .
$$

The same result is valid for $c=0$ and from numerical simulations for $0<c<1$ it seems to be valid in this region, too [18]. The reason such a result can appear is apparent from the transfer matrix calculations for $c=0$. In this case we have

$$
\rho_{N}(r, l)=\frac{c_{1}}{r^{2}} G\left(l / r^{2}\right)+c_{2} \varepsilon^{-3 / 2} r^{3}\left(2-l / r^{2}\right) \delta(l-\sqrt{3} \varepsilon),
$$

where $\varepsilon$ is a cut-off (the lattice spacing) and

$$
G(z)=\left(z^{-5 / 2}+\frac{1}{2} z^{-3 / 2}+\frac{14}{3} z^{1 / 2}\right) e^{-z}, \quad \text { for } \quad N \rightarrow \infty
$$

For low moments, $n=0$ and 1 , the terms in (33) which are singular for $l \rightarrow 0$ will dominate the evaluation of

$$
\left\langle l^{n}\right\rangle_{r, N}=\int_{\varepsilon} d l l^{n} \rho_{N}(r, l)
$$

while for $n \geq 2$ these terms become integrable and the cut-off dependence vanishes. It seems that the situation is the same at least for $c \in[-2,1)$. The exists a regular part, $\rho_{N}^{(\text {reg })}(r, l)$, of $\rho_{N}(r, l)$ such that

$$
d l \rho_{N}^{(\mathrm{reg})}(r, l)=d\left(l / r^{2}\right) G\left(l / r^{2}\right)
$$

is a function only of $l / r^{2}$, and which dominates the integral (35) for $n \geq 2$, while a part, singular for $l \rightarrow 0$, dominates (35) for $n=0$ and 1 .

In order to fully understand the concept of fractal dimension we still have to provide an explanation of an expression like (33) for $c \neq 0$.

\section{Acknowledgments}

J.A. acknowledges the support of the Professor Visitante Iberdrola grant and the hospitality at the University of Barcelona, where part of this work was done.

\section{References}

[1] H. Kawai, N. Kawamoto, T. Mogami and Y. Watabiki, Phys. Lett. B306, (1993) 19.

[2] J. Ambjørn and Y. Watabiki, Nucl.Phys.B445 (1995) 129.

[3] J. Ambjørn, K.N. Anagnostopoulos, U. Magnea and G. Thorleifsson, Geometrical interpretation of the KPZ exponents, to appear in Phys.Lett.B, e-Print Archive: hep-lat/9606012. 
[4] J. Ambjørn, J. Jurkiewicz and Y. Watabiki, Nucl.Phys.B454 (1995) 313.

[5] S. Catterall, G. Thorleifsson, M. Bowick and V. John, Phys. Lett. B354 (1995) 58.

[6] N. Kawamoto, V.A. Kazakov, Y. Saeki and Y. Watabiki, Phys. Rev. Lett. 68 (1992) 2113.

[7] V.A. Kazakov, I.K. Kostov and A.A. Migdal, Phys.Lett. 157B (1985) 295.

[8] J. Ambjørn, K.N. Anagnostopoulos, T. Ichihara, L. Jensen, N. Kawamoto, Y. Watabiki and K. Yotsuji, to appear.

[9] N. Kawamoto, Y. Saeki and Y. Watabiki, unpublished; Y. Watabiki, Progress in Theoretical Physics, Suppl. No. 114 (1993) 1; N. Kawamoto, In Nishinomiya 1992, Proceedings, Quantum gravity, 112, ed. K. Kikkawa and M. Ninomiya (World Scientific); In First Asia-Pacific Winter School for Theoretical Physics 1993, Proceedings, Current Topics in Theoretical Physics, ed. Y.M. Cho (World Scientific).

[10] Y. Watabiki, Fractal structure of space-time in two-dimensional quantum gravity, e-Print Archive: hep-th/9605185.

[11] J. Distler, Z. Hlousek and H. Kawai, Int. J. Mod. Phys. A5 (1990) 1093.

[12] N. Ishibashi and H. Kawai, Phys. Lett. B322 (1994) 67; B352 (1995) 75.

[13] J. Ambjørn, C.F. Kristjansen and Y. Watabiki, A String Field Theory for the $c=-2$ String, NORDITA-96/74P, TIT/HEP-353.

[14] G.K. Savvidy and N.G. Ter-Arutyunyan Savvidy, EPI-865-16-86 (1986); J. Comput. Phys. 97 (1991) 566.

[15] N.Z. Akopov, G.K. Savvidy and N.G. Ter-Arutyunyan Savvidy, J. Comput. Phys. 97 (1991) 573.

[16] M. Lüscher, Comput. Phys. Commun. 79 (1994) 100.

[17] F. James, Comput. Phys. Commun. 79 (1994) 111; Erratum 97 (1996) 357.

[18] J. Ambjørn and K.N. Anagnostopoulos, to appear. 